Nota técnica

\title{
Primer registro documentado de la Cigüeña Maguari (Ciconia maguari Gmelin, 1789; Aves: Ciconiidae) en Loreto, Perú
}

\author{
[First documented record of the Maguari Stork (Ciconia maguari, \\ Gmelin, 1789; Aves: Ciconiidae) in Loreto, Peru]
}
Luis Armando Garcia Solsol ${ }^{* 1}$, Gino Anthony Tuesta Cometivos ${ }^{1}$, Fredy Francisco Ramírez Arévalo², Anthony Giardenelli³ ${ }^{3}$ Juan Díaz Alván ${ }^{4}$

\author{
1. Universidad Nacional de la Amazonía Peruana (UNAP). Facultad de Ciencias Biológicas. \\ Pevas $5^{\text {ta }}$ cuadra, Iquitos, Maynas, Loreto, Perú. \\ Correos electrónicos: luisgarciasolsol@gmail.com (L. A. García * Autor por correspondencia), \\ ginoanthony29@gmail.com (G. A. Tuesta). \\ 2. Universidad Nacional de la Amazonía Peruana (UNAP). Facultad de Ciencias Forestales. \\ Pevas $5^{\text {ta }}$ cuadra, Iquitos, Maynas, Loreto, Perú. \\ Correo electrónico: fredy.ramirez@unapiquitos.edu.pe (F. F. Ramírez). \\ 3. Otorongo Expeditions. Nauta $3^{\text {ra }}$ cuadra, Iquitos, Maynas, Loreto, Perú. \\ Correo electrónico: giardea@yahoo.com (A. Giardenelli). \\ 4. Universidad Científica del Perú (UCP). Av. A. Quiñones km 2,5 (Iquitos), \\ San Juan Bautista, Maynas, Loreto, Perú. \\ Correo electrónico: jdiaz@ucp.edu.pe (J. Díaz).
}

\section{Resumen}

La Cigüeña Maguari (Ciconia maguari) se encuentra considerada en Perú como divagante, con avistamientos especialmente en la parte sureste del país. Documentamos la presencia de $C$. maguari en el Departamento de Loreto ampliando su distribución en Perú y proporcionamos algunos datos sobre su permanencia, comportamiento y número de individuos.

Palabras clave: Divagante, Nuevo registro, Ocurrencia en Perú.

\begin{abstract}
The Maguari Stork (Ciconia maguari) is considered in Peru as vagrant, with sightings especially in the southeastern part of the country. We document the presence of $C$. maguari in the Department of Loreto, expanding its distribution in Peru, and we provide some data on its permanence, behavior, and number of individuals.
\end{abstract}

Keywords: Vagrant, New record, Occurrence in Peru. 


\section{INTRODUCCIÓN}

La Cigueña Maguari (Ciconia maguari) es un ave de gran tamaño $(97-110 \mathrm{~cm})$, la única de su género en Sudamérica. Sus largas patas rojas y plumas blancas y negras, son características que lo hacen inconfundible (Elliot et al., 2020). Se distribuye principalmente al este de los Andes en Colombia, Venezuela, las Guayanas, norte de Brasil (Roraima, Amapá, Pará) y centro de Brasil (Mato Grosso), Bolivia, Uruguay y zona central de Argentina, río Negro (Elliot et al., 2020; Tavares y Siciliano, 2013; Luthin, 1987; Kahl, 1971). En Perú es divagante y muy rara en el sureste. Fue observada desplazándose solitaria o en parejas en los bosques de tierras bajas en Madre de Dios, así como a los 3650 msnm en los Andes peruanos (Schulenberg et al., 2010). Normalmente suelen encontrarse en campos abiertos en tierras bajas, no es común observarla en zonas boscosas. Se alimenta en sitios con presencia de cuerpos de agua dulce, incluyendo pastizales inundados, arrozales y pantanos (Elliot et al., 2020; Schulenberg et al., 2010). Busca alimento en aguas poco profundas, especialmente ranas y renacuajos, peces, anguilas, pequeños roedores acuáticos, serpientes, crustáceos e insectos acuáticos (Elliot et al., 2020; Tozetti et al., 2011). Está categorizada como de Preocupación Menor (LC) por la IUCN (2020), con una población que se mantiene estable en ausencia de evidencia de declives o amenazas sustanciales (Birdlife International, 2020).

Los primeros registros de la especie datan de junio de 1996, cuando Stotz et al. (2002) observa a $C$. maguari en el Santuario Nacional Pampas del Heath, Madre de Dios, posteriormente es incluido en la guía de campo de Aves de Perú de Clements y Shany (2001). Consecutivamente es avistada en distintos lugares, como los avistamientos no documentados llevadas a cabo por Rob Westerduijn (febrero 2004) en el río Amazonas, Loreto (M. A. Plenge, comunicación personal); río Madre de Dios, Madre de Dios (2003); río Tambopata, Madre de Dios (2005); río Manu, Madre de Dios (2005); Desaguadero, Puno (2005); Lago Titicaca, Puno (2010) y el
2011 en el río Heath, Madre de Dios (Angulo et al., 2017). En Perú la especie fue documentada por primera vez en agosto del 2003, un par de individuos fueron fotografiados por Roger Ahlman en la Laguna de Tanka (3876 msnm), Sicuani, departamento Puno (Angulo et al., 2017). Esto cambió su estatus de Hipotética a visitante no reproductiva en la lista de aves de Perú (Plenge, 2020; Angulo et al., 2017).

En esta nota científica se documenta por primera vez la presencia de la Cigüeña Maguari ( $C$. maguari) en el departamento Loreto, Perú, proporcionando algunos datos sobre permanencia, comportamiento y número de individuos de esta rara especie en Perú.

\section{REGISTROS}

El 15 agosto del 2020, L. A. García, G. A Tuesta y F. F. Ramírez observaron y fotografiaron a dos individuos adultos de $C$. maguari a las 16 horas y 15 minutos (Figura 1 y Figura 2), probablemente alimentándose a orillas del río Amazonas. Esto fue en la margen izquierda, cerca al puerto de Bellavista Nanay, donde confluyen los ríos Itaya, Nanay y Amazonas, distrito de Punchana, provincia de Maynas, departamento de Loreto (Tabla 1 y Figura 1). Estos individuos al percatarse de nuestra presencia se desplazaron con rumbo desconocido hacia el interior de los sembríos de arroz (Oryza sativa).

El 17 de agosto del 2020, J. Díaz y A. Giardenelli observaron y fotografiaron a 6 individuos de $C$. maguari a las 7:00 horas, a $4 \mathrm{~km}$ de distancia del registro del 15 de agosto del 2020. Este grupo fue observado buscando alimento a orillas del río Amazonas. Algunos individuos fueron fotografiados alimentándose de peces muertos que probablemente fueron dejados por pescadores artesanales (Figura 3).

El 20 de agosto del 2020, A. C. Taricuarima y L. A. García observaron y fotografiaron a las 9:00 horas a 4 individuos de $C$. maguari en los alrededores de Barrio Florido, Distrito de Punchana, Provincia de Maynas, Departamento de Loreto 
Tabla 1. Registros de la Cigüeña Maguari (Ciconia maguari) en Loreto, Perú.

\begin{tabular}{|c|c|c|c|c|c|c|c|c|}
\hline $\mathbf{N}^{\circ}$ & Observadores & $\begin{array}{l}\mathrm{N}^{\circ} \text { de } \\
\text { indivi- } \\
\text { duos }\end{array}$ & Fechas & $\begin{array}{c}\text { Hora de } \\
\text { Observ. } \\
(24 \text { h) }\end{array}$ & $\begin{array}{l}\text { Lugar de ob- } \\
\text { servación }\end{array}$ & $\begin{array}{c}\text { Docu- } \\
\text { mentado } \\
\text { (Foto- } \\
\text { grafía) }\end{array}$ & Coordenadas & $\begin{array}{l}\text { Eleva- } \\
\text { ción } \\
\text { (msnm) }\end{array}$ \\
\hline 1 & $\begin{array}{l}\text { Luis A. Garcia } \\
\text { Solsol }\end{array}$ & 1 & $15 / 08 / 2020$ & $16: 15$ & $\begin{array}{l}\text { Río Amazonas, } \\
\text { Loreto }\end{array}$ & $\mathrm{Si}$ & $03^{\circ} 41^{\prime} 52^{\prime \prime} \mathrm{S} ; 73^{\circ} 14^{\prime} 09^{\prime \prime} \mathrm{W}$ & 86 \\
\hline 2 & Juan Díaz Alván & 6 & $17 / 08 / 2020$ & 7:00 & $\begin{array}{l}\text { Río Amazonas, } \\
\text { Loreto }\end{array}$ & $\mathrm{Si}$ & $03^{\circ} 43^{\prime} 23^{\prime \prime} \mathrm{S} ; 73^{\circ} 12^{\prime} 10^{\prime \prime} \mathrm{W}$ & 85 \\
\hline 3 & $\begin{array}{l}\text { Avita Taricuarima } \\
\text { Dreyfus }\end{array}$ & 4 & $20 / 08 / 2020$ & 9:00 & $\begin{array}{l}\text { Barrio Florido, } \\
\text { Loreto }\end{array}$ & $\mathrm{Si}$ & $03^{\circ} 42^{\prime} 19^{\prime \prime} \mathrm{S} ; 73^{\circ} 14^{\prime} 18^{\prime \prime} \mathrm{W}$ & 85 \\
\hline 4 & $\begin{array}{l}\text { Luis A. Garcia } \\
\text { Solsol }\end{array}$ & 5 & $29 / 08 / 2020$ & $8: 20$ & $\begin{array}{l}\text { Río Amazonas, } \\
\text { Loreto }\end{array}$ & $\mathrm{Si}$ & $03^{\circ} 42^{\prime} 47^{\prime \prime} \mathrm{S} ; 73^{\circ} 13^{\prime} 06^{\prime \prime} \mathrm{W}$ & 85 \\
\hline 5 & $\begin{array}{l}\text { Luis A. Garcia } \\
\text { Solsol }\end{array}$ & 6 & $10 / 09 / 2020$ & $7: 45$ & $\begin{array}{l}\text { Río Amazonas, } \\
\text { Loreto }\end{array}$ & $\mathrm{Si}$ & $03^{\circ} 38^{\prime} 56^{\prime \prime} \mathrm{S} ; 73^{\circ} 12^{\prime} 25^{\prime \prime} \mathrm{W}$ & 85 \\
\hline 6 & $\begin{array}{l}\text { Luis A. Garcia } \\
\text { Solsol }\end{array}$ & 3 & $22 / 09 / 2020$ & $8: 50$ & $\begin{array}{l}\text { Río Amazonas, } \\
\text { Loreto }\end{array}$ & $\mathrm{Si}$ & $03^{\circ} 43^{\prime} 19^{\prime \prime} \mathrm{S} ; 73^{\circ} 12^{\prime} 29^{\prime \prime} \mathrm{W}$ & 85 \\
\hline 7 & $\begin{array}{l}\text { Luis A. Garcia } \\
\text { Solsol }\end{array}$ & 4 & $2 / 10 / 2020$ & $9: 10$ & $\begin{array}{l}\text { Río Amazonas, } \\
\text { Loreto }\end{array}$ & $\mathrm{Si}$ & $03^{\circ} 42^{\prime} 39^{\prime \prime} \mathrm{S} ; 73^{\circ} 13^{\prime} 17^{\prime \prime} \mathrm{W}$ & 85 \\
\hline 8 & $\begin{array}{l}\text { Luis A. Garcia } \\
\text { Solsol }\end{array}$ & 7 & $15 / 10 / 2020$ & $7: 30$ & $\begin{array}{l}\text { Río Amazonas, } \\
\text { Loreto }\end{array}$ & $\mathrm{Si}$ & $03^{\circ} 41^{\prime} 48^{\prime \prime} \mathrm{S} ; 73^{\circ} 14^{\prime} 31^{\prime \prime} \mathrm{W}$ & 85 \\
\hline
\end{tabular}

Fuente: Elaboración propia.

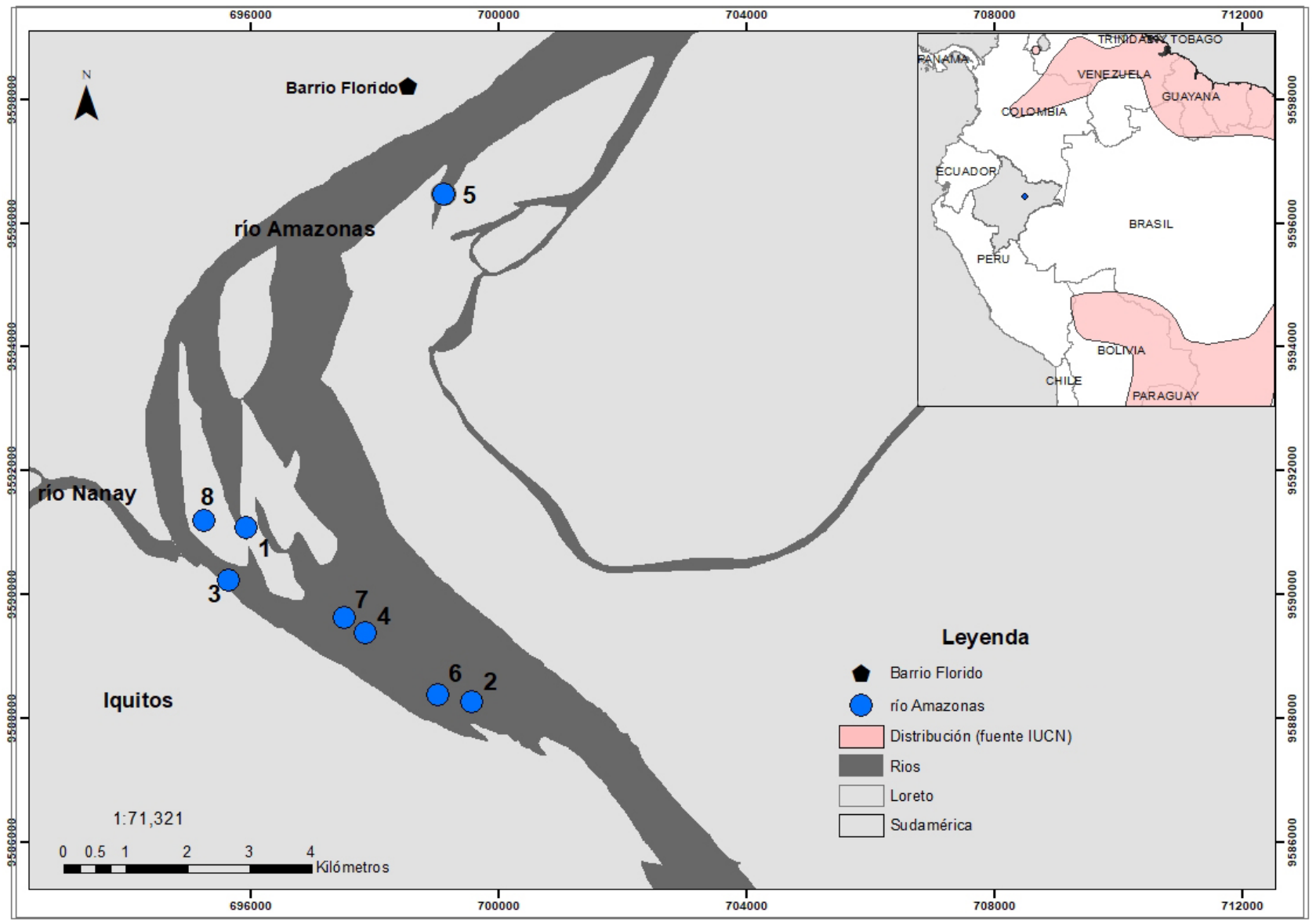

Figura 1. Mapa de registros de la Cigüeña Maguari (Ciconia maguarı) en Loreto, Perú 


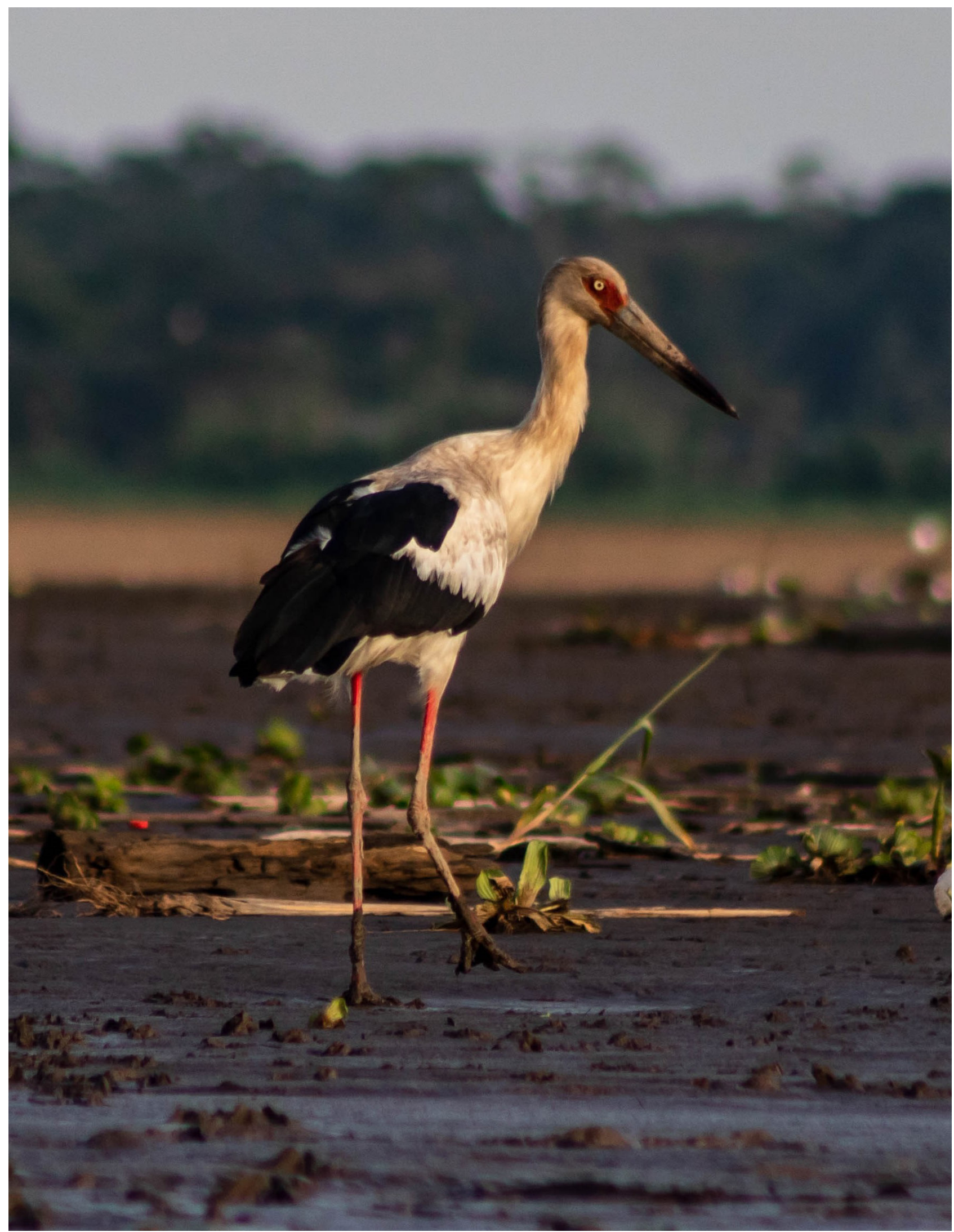

Figura 2. Individuo adulto de Cigüeña Maguari (Ciconia maguarı) observada el 15 de agosto del 2020 a orillas del río Amazonas, Loreto, Perú. Foto: Luis A. Garcia. 


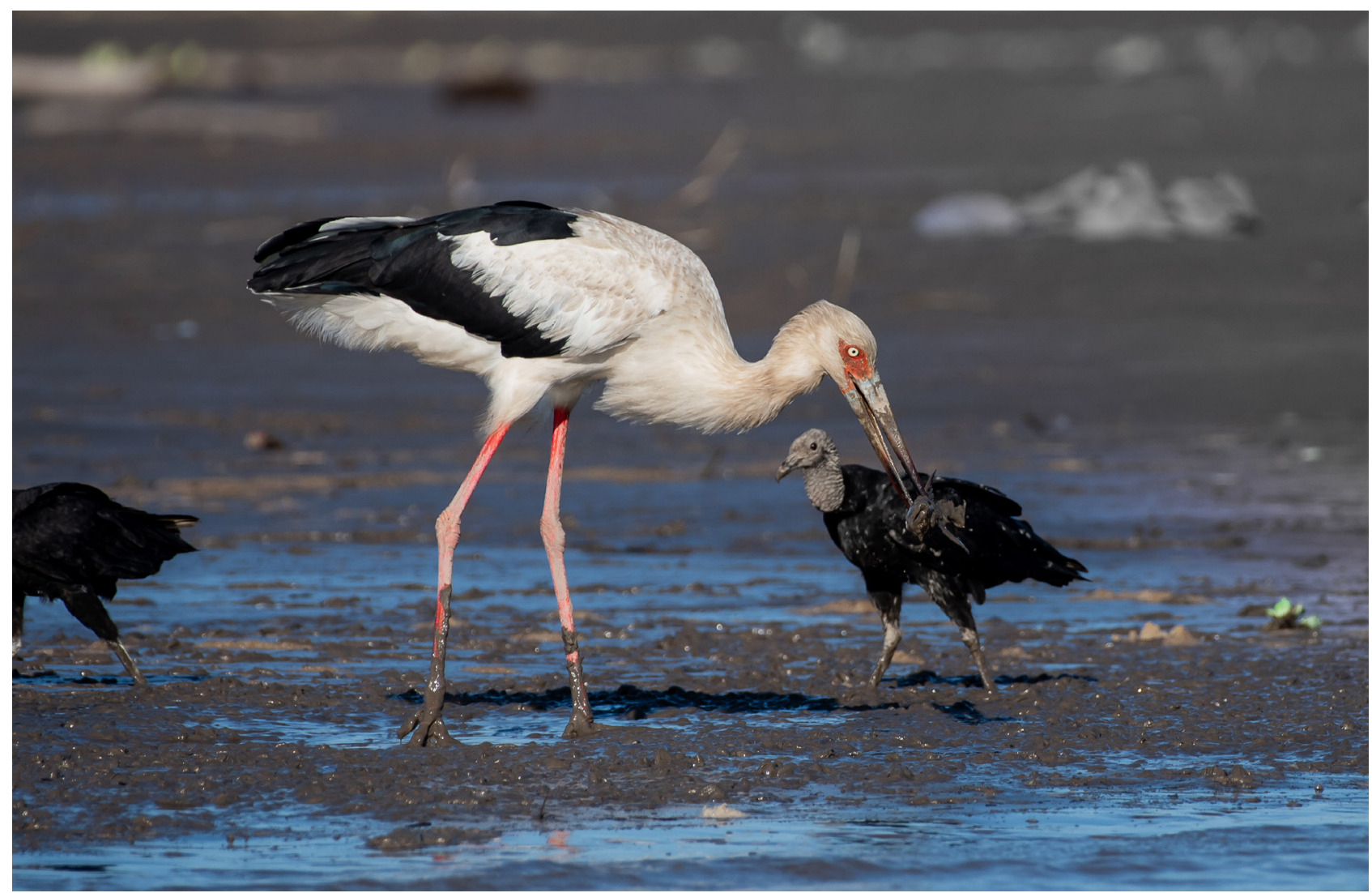

Figura 3. Individuo de Cigüeña Maguari (Ciconia maguari), alimentándose de peces junto a Gallinazos de Cabeza Negra (Coragyps atratus) a orillas del río Amazonas, Loreto, Perú. Foto: A. Giardanelli.

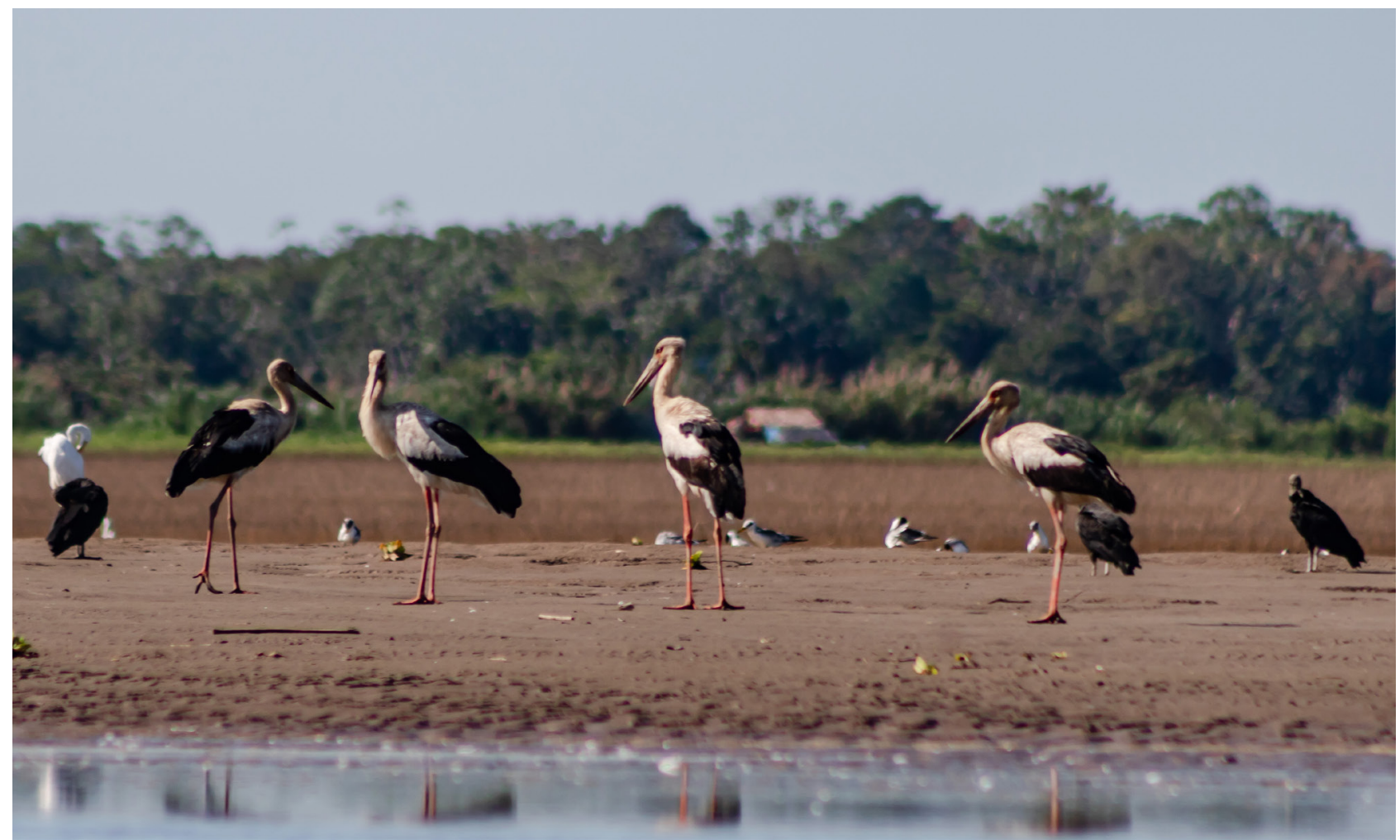

Figura 4. Individuos de Cigüeña Maguari (Ciconia maguarı), observados a orillas del río Amazonas, Loreto, Perú. Foto: A. Taricuarima. 
(Tabla 1, Figura 1), a $8 \mathrm{~km}$ de distancia del puerto de Bellavista Nanay, aguas abajo del río Amazonas. Se encontraban buscando alimento a orillas del río, junto a algunos individuos de Garza Grande (Ardea alba), Garcita Blanca (Egretta thula) y Gallinazos de Cabeza Negra (Coragyps atratus).

El 29 de agosto del 2020, L. A. Garcia y G. A. Tuesta observaron a las 8:20 horas a 5 individuos de $C$. maguari en los alrededores del encuentro de los ríos Itaya y Amazonas. Algunos de estos individuos se encontraban disputándose el alimento con algunos individuos de $C$. atratus, probablemente peces en descomposición (Figura 4).

El 10 de setiembre del 2020 a las 7:45 horas, L. A. Garcia observó y fotografió 6 individuos de $C$. maguari a orillas del río Amazonas. Se encontraban alimentándose de peces descartados por los pescadores y probablemente de otros pequeños animales atrapados en el lodo. El mismo comportamiento fue observado el 22 de setiembre, el 2 y 15 de octubre del 2020, variando el número de individuos entre 3, 4 a 7 respectivamente, pero siempre ubicados entre la boca de los ríos Nanay, Itaya, aguas abajo y arriba del río Amazonas, no sobrepasando los $8 \mathrm{~km}$ de distancia de la zona de encuentro de estos ríos. Algunos otros registros posteriores a nuestro avistamiento en esta misma zona se encuentran reportados en la plataforma de eBird.

\section{DISCUSIÓN}

Los registros presentados confirman la presencia de $C$. maguari en el departamento de Loreto, Perú. No sabemos exactamente desde donde estaría llegando, pero considerando el mapa de distribución de la especie en Sudamérica (Figura 1), posiblemente sea desde el suroeste colombiano o se trataría de individuos errantes que podrían estar dispersándose a lo largo del río Amazonas, ya que existen registros en áreas cercanas a Manaos, Brasil, llevadas a cabo por G. Leite en julio del 2020 (ebird.org/checklist/ S71330347). Otra opción es que provengan desde el sur del Perú, ya que es conocida la gran capacidad de desplazamiento de la especie (Elliot et al., 2020; Olaciregui et al., 2010), especialmente por requerimientos de alimento y hábitats adecuados.

De acuerdo con nuestras observaciones, la especie se estaría desplazando en búsqueda de hábitats favorables para su establecimiento temporal, como son los espacios abiertos, áreas cercanas a ríos o cochas, arrozales, pastos inundables y por la disponibilidad de alimento (Elliot et al., 2020; Olaciregui et al., 2014). Esto se puede notar a lo largo del río Amazonas y probablemente en otras áreas en Loreto, tal como se conoce para otras especies recientemente registradas que se benefician de estos nuevos espacios como resultado de actividades antropogénicas, entre ellas se encuentra el Ibis Escarlata Eudocimus ruber (Ruiz-Ramos et al., 2020); el Zambullidor Menor Tachybaptus dominicus (Ruiz-Ramos et al., 2018); el Ibis de Cara Pelada Phimosus infuscatus (Socolar et al., 2018); la Calandria Tropical Mimus gilvus (Cuelo, 2018); el Avefría Tero Vanellus chilensis (Ruiz-Ramos et al., 2017); o el Caracara Carancho Caracara plancus (Piana et al., 2012).

En cuanto al número de individuos, el grupo observado desde agosto hasta mediados de octubre llega a estar conformado por un máximo de 7 individuos. Esto es diferente a lo que menciona Schulenberg et al. (2010): individuos solitarios o en parejas que divagan. Si las observaciones se hacen más constantes durante los próximos años la especie podría ser un visitante regular en la zona, especialmente en época de vaciante, cuando el nivel del agua del río está bajo, probablemente por los hábitats favorables que encuentra. Los individuos observados no se mostraron temerosos ante la presencia de pescadores, ya que en algunas ocasiones conseguían alimento cuando dejaban peces en los alrededores o de otros animales, como se mencionan en otros reportes (Elliot et al., 2020; Tozetti et al., 2011). Por otro lado, se sabe que la época reproductiva de $C$. maguari es entre los meses de junio y noviembre de cada año, especialmente en los llanos venezolanos y zo- 
nas del sur de Brasil y norte de Argentina (Elliot et al., 2020; González, 1998; Thomas, 1984, 1986; Kahl, 1971). En nuestras observaciones (agosto-octubre) no registramos ningún comportamiento reproductivo en los individuos observados. Por lo tanto, podemos concluir que $C$. maguari estaría visitando la selva de Loreto por la presencia de hábitats favorables y alimento que encuentra en la zona y quizás en un futuro sea más constante su registro.

\section{AGRADECIMIENTOS}

A Manuel A. Plenge por la valiosa información proporcionada de $C$. maguari. A Surech A. Ruiz-Ramos por la elaboración del mapa de distribución de la especie. A Fernando Angulo y un revisor anónimo por las sugerencias que mejoraron el manuscrito. A IUCN por el uso del mapa de distribución de $C$. maguari.

\section{REFERENCIAS BIBLIOGRÁFICAS}

Angulo, F., Begazo, A., Lane, D., Plenge, M., Stotz, D., Ugarte, M., Valqui, V. y Walker, B. (2017) Reporte del Comité de Registros de Aves Peruanas (CRAP) del periodo 2016. Boletín de la Unión de Ornitólogos del Perú (UNOP), 12 (2), 49-56.

BirdLife International. (2020) Species factsheet: Ciconia maguari [en línea], Disponible en <http://datazone.birdlife.org/species/ factsheet/maguari-stork-ciconia-maguari/ text>. [Consulta: 10/09/2020].

Clements, J. y Shany, N. (2001) A field guide to the birds of Peru. Temecula: Ibis Publishing Company.

Cuelo P., W. (2018) Primer registro documentado de Calandria Tropical (Mimus gilvus) para el Perú. Boletín de la Unión de Ornitólogos del Perú (UNOP), 13 (1), 7-9.

eBird. (2020) Una base de datos en línea para la abundancia y distribución de las aves. [en línea], Disponible en <https://ebird.org/ home> [Consulta: 26 de setiembre 2020].

Elliott, A., Boesman P.F.D. y Kirwan G. M. (2020) Maguari Stork (Ciconia maguarı), versión 1.0. En Birds of the World (J. del Hoyo, A. Elliott, J. Sargatal, DA Christie y E. de Juana, Editores). Cornell Lab of Ornithology, Ithaca, NY, USA. [en línea], Disponible en <https://doi.org/10.2173/ bow.magsto1.01> [Consulta: 20 setiembre 2020].

González, J. A. (1998) Phenology and reproductive success of the Maguari Stork in the Southern Llanos of Venezuela. Colonial Waterbirds. 21, 135-142.

IUCN. 2020. The IUCN Red List of Threatened species. (2020) Versión 2020-1. Disponible en: <https://www.iucnredlist.org. Accedido el 20/06/2020.> [Consulta: 18 setiembre 2020].

Olaciregui, C., Quevedo, A., Bartels, A., Ortiz, E., Fernández, M. A. y Avila, A. (2014) First records of Maguari Stork Ciconia maguari in north-western South America. Cotinga, 36, 113-115.

Kahl, M. P. (1971) Observations on the Jabiru and Maguari Storks in Argentina, 1969. The Condor, 73, 220-229.

Luthin, C. S. (1987) Status and conservation priorities for the world's stork species. CoIonial Waterbirds 10, 181-202.

Piana, R. P., Díaz, A. J., Chalco L., J. J., Sevillano, C. S., Saboya del Castillo, P., Cubas P.S. (2012) El Caracara Carancho (Caracara plancus) estaría expandiendo su rango de distribución hacia el norte y oeste de la Amazonía peruana. Boletín de la Unión de Ornitólogos del Perú (UNOP), 7 (2), 5-11.

Plenge, M. (2020) Lista de aves del Perú. Boletín de la Unión de Ornitólogos del Perú. [en línea], Disponible en <https://sites.google. com/site/boletinunop/checklist. > [Consulta: 18 setiembre 2020].

Ruiz-Ramos, S. A., Alegría-Torres, B., Ayapi-Da-Silva, J. A y Díaz-Alván, J. (2017) Registro documentado del Avefría Tero (Vanellus chilensis) en selva norte del Perú. Boletín de la Unión de Ornitólogos del Perú (UNOP), 12 (1), 15-18.

Ruiz-Ramos, S. A., Ayapi-Da-Silva, J. A., Alegría-Torres, B., y Díaz, J. (2018) Registro documentado del Zambullidor Menor (Tachybaptus dominicus) en la selva norte de 
Loreto. Boletín de la Unión de Ornitólogos del Perú (UNOP), 13 (2), 12-15.

Ruiz-Ramos, S. A, Angulo-Perez, N. C., Saboya, P., Gaviria, J. L. y Díaz, J. (2020) Registros documentados del Corocoro Escarlata (Eudocimus ruber Linnaeus, 1758; Aves: Threskiornithidae) en Loreto, Perú. Ciencia Amazónica, 8 (1), 123-130.

Schulenberg, T. S., Stotz, D. F., Lane, D. F., O'Neill, J. P. y Parker III, T. A. (2010) Birds of Peru. Revised and updated edition. Princeton, NJ: Princeton University Press.

Socolar, J. B., Díaz-Alván. J., Saboya, P., Pomara, L. Y., O'Shea, B. J., Cubas P. S., Stotz, D., Schmitt, F., Graham, D., Carnes, B. H. y Ruelas, I. E. (2018) Noteworthy bird records from northeastern Peru reveal connectivity and isolation in the western Amazonian avifauna. The Wilson Journal of Ornithology, 130, 94-111.

Stotz, D. F., Montambault, J. R., Pequeño, T., Valdez, A., Mack, A. y Quiroga, C. (2002) Evaluación de la avifauna de Pampas del Heath, Perú y Alto Madidi, Bolivia. En Informes de las evaluaciones biológicas de Pampa del Heath, Perú, Alto Madidi, Bolivia, y Pando, Bolivia. Conservation International, RAP Bulletin of Biological Assessment, 24: 49.
Tavares, D. C. y Siciliano, S. (2013) Scientific Note Notes on records of Ciconia maguari (Gmelin, 1789)(Aves, Ciconiidae) on northern Rio de Janeiro State, Southeast Brazil. Pan-American Journal of Aquatic Sciences, 8(4), 352-357.

Thomas, B. T. (1984) Maguari Stork nesting: juvenile growth and behavior. Auk, 101, 812-823.

Thomas, B. T. (1986) The behavior and breeding of adult Maguari Storks. The Condor, 88, 26-34.

Tozetti, A. M., Fontana, C. S., Oliveira, R. B., Pontes, G. M. F. (2011) Diet of a Maguari Stork (Ciconia maguari, Aves, Ciconiidae) in southern Brazil: the opportunist predation of snake like preys?. Pan-American Journal of Aquatic Sciences, 6, 65-67.

\section{Conflicto de interés}

Los autores declaramos no tener ningún conflicto de interés. 